\title{
Apparent diffusion coefficient measurements of the pancreas, pancreas carcinoma, and mass-forming focal pancreatitis
}

\section{Philipp Wiggermann', Robert Grützmann², Angelika Weissenböck ${ }^{3}$, Peter Kamusella ${ }^{4}$, Dag-Daniel Dittert ${ }^{5}$ and Christian Stroszczynski ${ }^{1}$}

${ }^{1}$ University Medical Center Regensburg, Institute of Diagnostic Radiology, Regensburg; ${ }^{2}$ University Hospital Carl Gustav Carus Dresden, Department of Visceral, Thoracic and Vascular Surgery, Dresden; ${ }^{3}$ University Hospital Carl Gustav Carus Dresden, Dresden; ${ }^{4}$ University Hospital Carl Gustav Carus Dresden, Department of Radiology, Dresden; ${ }^{5}$ University Hospital Carl Gustav Carus Dresden, Department of Pathology, Dresden, Germany

Correspondence to: Philipp Wiggermann. Email: philipp.wiggermann@ukr.de

\begin{abstract}
Background: Mass-forming focal pancreatitis (FP) may mimic pancreatic cancer (PC) on magnetic resonance (MR) imaging, and the preoperative differential diagnosis is often difficult. Recently, the usefulness of diffusion-weighted imaging (DWI) in the diagnosis of pancreatic cancer has been reported in several studies.

Purpose: To investigate if apparent diffusion coefficient (ADC) measurements based on diffusion-weighted echo-planar imaging (DW-EPI) may distinguish between normal pancreas parenchyma, mass-forming focal pancreatitis, and pancreas carcinoma.

Material and Methods: MRI was performed on 64 patients: 24 with pancreas carcinoma (PC), 20 with massforming focal pancreatitis (FP), three patients with other focal pancreatic disease as well as 17 controls without any known pancreatic disease. Diffusion-weighted sequence with ADC maps and T2-weighted sequence for anatomical information was performed. Apparent diffusion coefficient (ADC) maps were automatically created and analyzed using a dedicated user interface. In the group with pancreas disease the abnormal parenchyma was detected by using T1- and T2-weighted images and the region of interest (ROI) was transferred exactly to the ADC map and the coefficients were registered. In the control group the ROI was set to the head of the pancreas followed by a similar registration of the ADCs.

Results: ADC values for mass-forming FP and PC differed significantly from ADC values for normal pancreas parenchyma $(P=0.001 / P=0.002)$. Mean ADC values for mass-forming FP were $0.69 \pm 0.18 \times 10^{-3} \mathrm{~mm}^{2} / \mathrm{s}$. $A D C$ values for $P C$ were $0.78 \pm 0.11 \times 10^{-3} \mathrm{~mm}^{2} / \mathrm{s}$, compared to ADC values of $0.17 \pm 0.06 \times 10^{-3} \mathrm{~mm}^{2} / \mathrm{s}$ in the control group. However there was no significant difference in ADCs between PC and mass-forming FP $(P=0.15)$.

Conclusion: ADC measurements clearly differentiated between normal pancreatic tissue and abnormal pancreas parenchyma (PC and mass-forming FP). However there is an overlap in values of PC and mass-forming FP, with the consequent problem of their correct identification.
\end{abstract}

Keywords: MRI, diffusion-weighted imaging, apparent diffusion coefficient, abdominal imaging, pancreas

Submitted December 7, 2009; accepted for publication November 23, 2011

Magnetic resonance imaging (MRI) along with MR cholangiopancreatography (MRCP) have become the imaging modalities of choice to asses both the structure and the function of the pancreas. In addition, diffusion-weighted imaging (DWI) has been shown to provide useful information to discriminate between normal pancreas, pancreatitis, and pancreatic cancer (1). Diffusion-weighted MRI and apparent diffusion coefficient (ADC) measurements have 
been applied to the abdomen (2-5). The results suggest that ADC measurements can be useful in the evaluation of diffuse liver $(6)$ and renal diseases $(7,8)$ as well as for characterizing focal hepatic $(4,9)$, pancreatic (10-15), and renal (16) lesions. The purpose of our study was to evaluate DWI findings and measure the difference between ADC values of normal pancreas parenchyma, mass-forming focal pancreatitis (FP) and pancreas carcinoma (PC). We chose these pathologic conditions, as differentiation between them can be difficult. As there is a wide range of reported ADC values $(10,17,18)$ for PC and mass-forming FP we had to establish our own reference values. Our aim was to test whether there is a significant difference in ADCs for normal pancreas and abnormal pancreas parenchyma (PC and mass-forming FP), as well as to test whether or not it is possible to differentiate between PC and massforming FP by using ADC measurements. This would allow us to establish a non-invasive imaging of pancreatic lesions protocol in the clinical routine to differentiate between PC and mass-forming FP.

\section{Material and Methods}

\section{Patients}

In this prospective study 64 subjects were included. Informed consent was obtained from all participants. The PC group was composed of 24 patients (12 women and 12 men; age range 38-81 years, mean age 64 years) with histological proof of ductal adenocarcinoma (PC) through Whipple procedure. Twenty patients $(7$ women and 13 men, age range 15-74 years, mean age 52 years) were included in the mass-forming FP group. Sixteen of these patients underwent surgery because malignancy could not be ruled out by either imaging or fine-needle aspiration. In four patients mass-forming FP was diagnosed without histopathologic proof, based on repeated imaging (MRI or dynamic computed tomography) and clinical follow-up (13-25 months) after the initial presentation. Seventeen subjects without any pancreatic disease (5 women and 12 men; age range 12-78 years, mean age 45 years) were included as control group and had normal MRI/MRCP findings.

All patients underwent the same imaging protocol for the diffusion-weighted imaging, and were scanned within 14 days prior to the surgery. Histological findings were determined from the records of our Department of Pathology. We had to exclude three patients from our study due to the following conditions: serous cyst adenoma (1), adenocarcinoma of unknown primary localization (1), and diffuse disease of intestine (measurement not possible) (1).

\section{MR protocol}

Diffusion-weighted sequence with ADC maps as well as the routine pancreatic MR imaging protocol for anatomical information were performed using a Magnetom Avanto $1.5 \mathrm{~T}$ scanner (Siemens Medical Solutions, Erlangen, Germany) and a commercially available cp body phased array coil. Diffusion-weighted images were acquired using a single-shot echo-planar imaging pulse sequence in expiration breath-hold. The following parameters were used for the axial DW-EPI sequence: matrix size of $144 \times$ 192, a field-of-view of $375 \times 500$ (pixel size $2.6 \times 2.6 \mathrm{~mm}$ ), and a section thickness of $2.6 \mathrm{~mm}$. The $\mathrm{b}$ factors used were 50 and $500 \mathrm{~s} / \mathrm{mm}^{2}$. In addition the routine pancreatic MR imaging protocol for the patient group consisted of TRUE-FISP imaging sequences, non-contrast T1-weighted fat-suppressed and dynamic gadolinium-enhanced gradientecho imaging as well as magnetic resonance cholangiopancreatography (MRCP) and angiography (MRA). The ADC maps were automatically created and analyzed using a dedicated user interface (Leonardo, Siemens Medical Solutions, Erlangen, Germany). The pancreas lesions were detected using the T1- and T2-weighted images and the ROI was transferred exactly to the ADC map and the according coefficients were registered (Figs. 1 and 2). In the control group the ROI was set to the head of the pancreas gland followed by a similar registration of the ADCs. In the diseased population ROIs ranged from 70 to $879 \mathrm{~mm}^{2}$
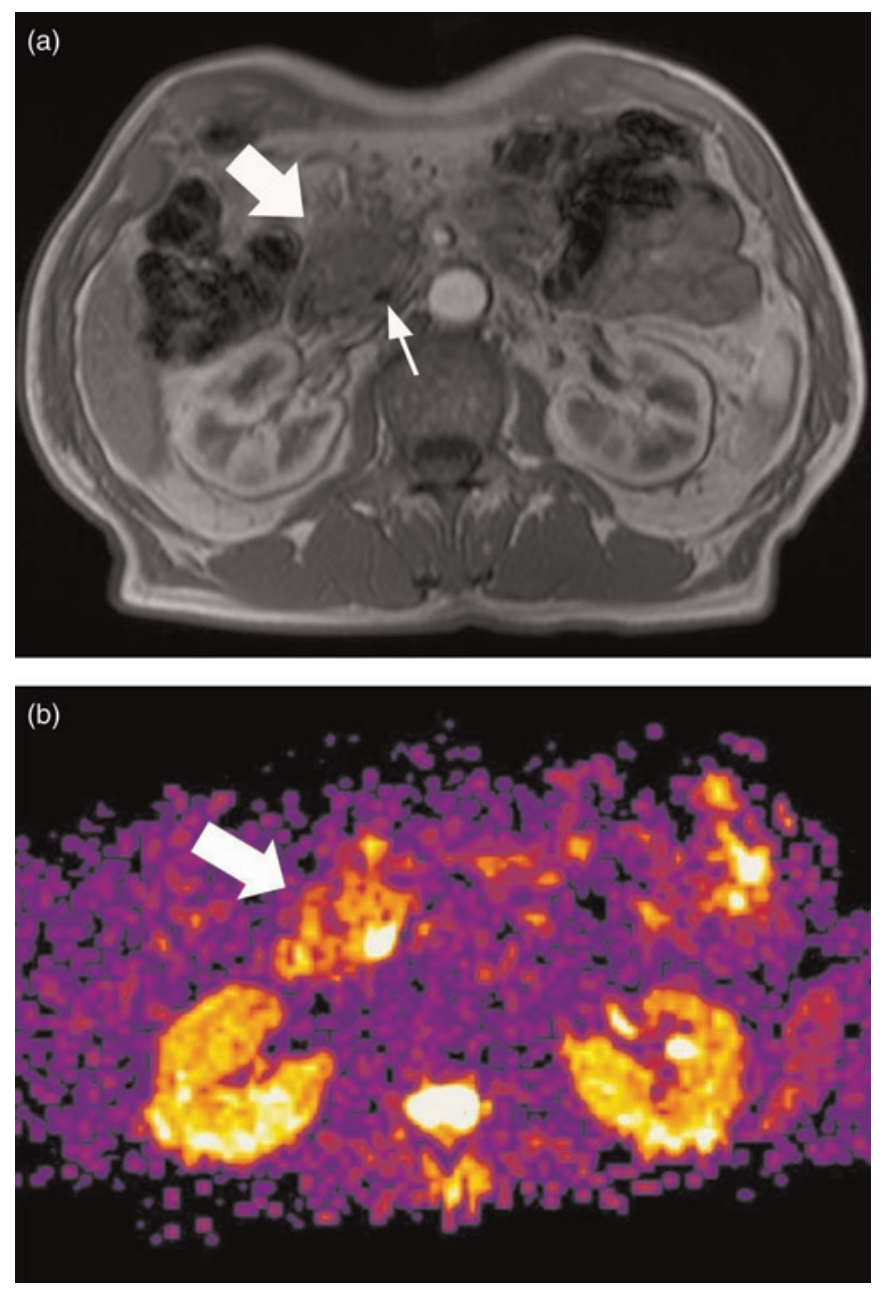

Fig. 1 A 69-year-old patient with a pancreatic adenocarcinoma of the head. (a) Dynamic post-contrast pancreatic phase T1-weighted axial MR image, the lesion shows heterogeneous hypo enhancing signal (large arrow), good depiction of the slightly dilated main pancreatic duct (small arrow); (b) Axial ADC map calculated from basis images obtained with $b$ values of $50,500 \mathrm{~s} / \mathrm{mm}^{2}$ in the same patient clearly shows the lesion. On the ADC map, higher diffusion is depicted as higher signal intensity 

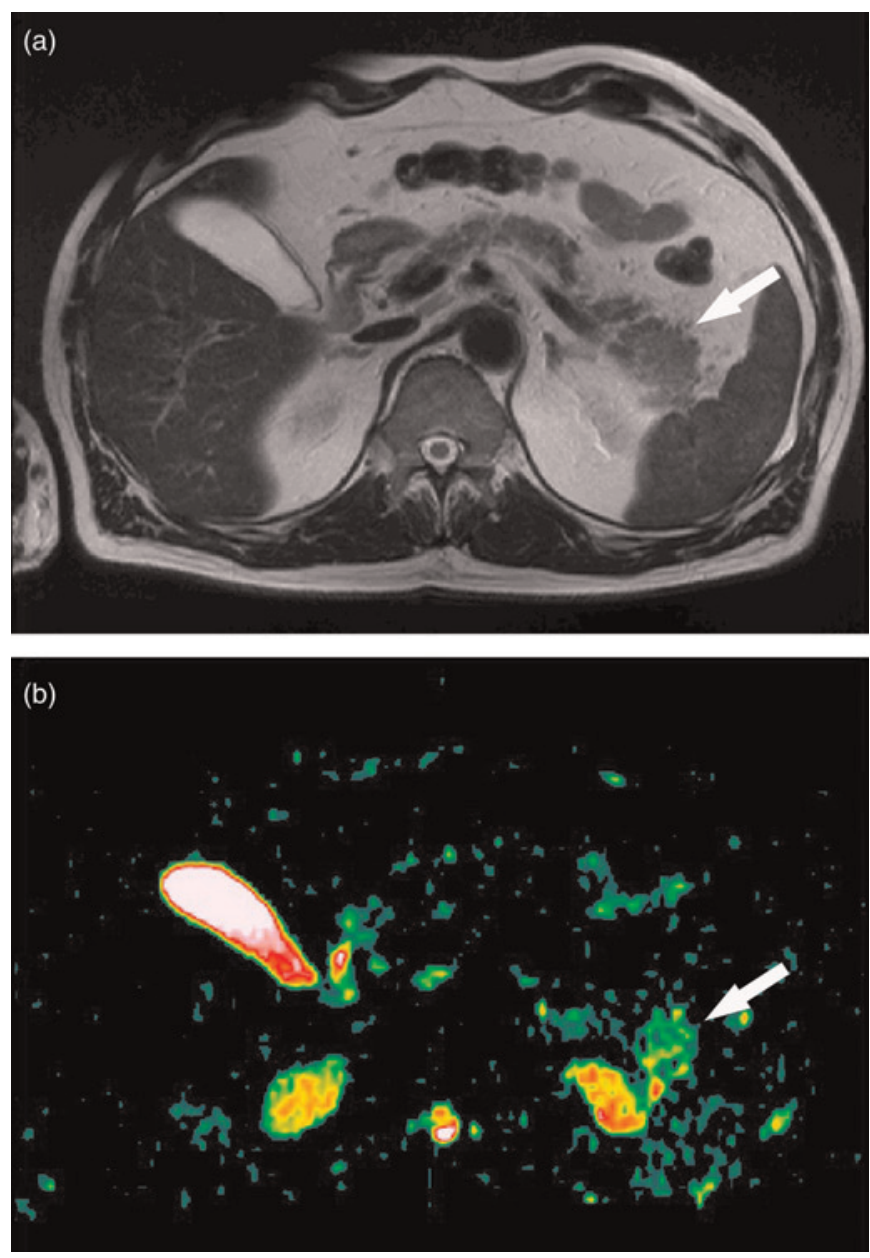

Fig. 2 A 56-year-old patient with mass-forming focal pancreatitis of the tail. (a) T2-weighted axial MR image; (b) Axial ADC map calculated from basis images obtained with $b$ values of $50,500 \mathrm{~s} / \mathrm{mm}^{2}$ in the same patient clearly shows the lesion. On the ADC map, higher diffusion is depicted as higher signal intensity

(mean $354 \mathrm{~mm}^{2}$ ), whereas in the control group a fixed ROI of $100 \mathrm{~mm}^{2}$ was used.

\section{Image analysis}

Consensus reading was performed by two experienced radiologists (15 years and 6 years of experience). ADCs were calculated on a workstation with dedicated software. Signal intensities on ADC maps were measured by using an operator-defined region of interest (ROI). ADCs were calculated for normal pancreas and focal lesions of the pancreas. The ROIs for the pancreas were circular. Pancreas vessels, the pancreatic duct and the common bile duct were left out of the ROI. At the time of ROI placement, the operator was aware of the location of the lesions and could refer to routine MR images and to the histopathological diagnosis. We examined patients with PC, mass-forming FP, and without pancreatic disease. In three patients with massforming FP and pancreatic pseudo cysts the ROI was placed outside these cystic lesions. ADC values of the control group were used to establish the normal values. The ROI within each lesion was circular and placed on the largest possible area, including necrotic parts. Mean ADCs of the two lesion types and mean values of healthy pancreatic tissue were compared.

\section{Statistical analysis}

The non-parametric Mann-Whitney $U$ test was used to determine whether there was a significant difference between ADC values for normal pancreas, PC, and massforming FP. The degree of inter-observer agreement in ADC value measurements was evaluated using the interclass correlation (ICC) coefficient. An ICC value of less than 0.40 indicates poor reproducibility, ICC values between 0.40 to 0.75 indicate a fair to good reproducibility, and an ICC value of greater than 0.75 shows excellent reproducibility. All values were expressed as mean $\pm \mathrm{SD}$, and a $P$ value of $<0.05$ was considered statistically significant. All statistical analysis was performed using SPSS for Windows, version 15.0 (SPSS Inc., Chicago, IL, USA).

\section{Results}

ADC measurements for all 24 PCs as well as the 20 massforming FP showed lower ADC values compared to normal pancreas parenchyma. The maximum tumor diameter of PC ranged from 1.7 to $6.1 \mathrm{~cm}$ (mean $3.8 \mathrm{~cm}$ ), the corresponding values for the mass-forming $\mathrm{FP}$ where 2.2 to $5 \mathrm{~cm}$ (mean $3.4 \mathrm{~cm}$ ).

The corresponding ADC values $\left(\times 10^{-3} \mathrm{~mm}^{2} / \mathrm{s}\right)$ were as follows: pancreatic carcinoma $(n=24), \quad 0.78 \pm 0.11$. Mass-forming FP $(n=20), 0.69 \pm 0.18$, and normal pancreas in healthy volunteers $(n=17), 0.17 \pm 0.06$ (Table 1 , Fig. 3).

ADC values of the pancreatic carcinoma proved to be significantly higher compared with those of the normal pancreatic tissue in healthy volunteers (Table 2). The Mann-Whitney $U$ test demonstrated a statistically significant difference $(P<0.001)$. ADC values of the mass-forming FP also proved to be significantly higher compared with those of the normal pancreatic tissue. The Mann-Whitney $\mathrm{U}$ test demonstrated a statistically significant difference compared with those of the normal pancreatic tissue albeit at a lower significance level $(P<0.002)$. There was no significant difference in ADCs between PC and mass-forming FP $(P=0.15)$. The inter-observer agreement for ADC measurements was analyzed using the ICC method. A 'fair to good agreement' was obtained for the average ADC value of mass-forming FP (ICC $=0.623)$. 'Excellent' inter-observer agreement was observed for the ADC values of PC and the control group (ICC $=0.768$ and ICC $=0.823$ ).

Table 1 Mean ADC values for normal pancreatic tissue, pancreatic carcinoma, and mass-forming focal pancreatitis

\begin{tabular}{lll}
\hline Pancreatic disease & $\boldsymbol{n}$ & ADC values \pm S.D.* \\
\hline None & 17 & $0.17 \pm 0.06$ \\
Pancreatic carcinoma & 24 & $0.78 \pm 0.11$ \\
Mass-forming FP & 20 & $0.69 \pm 0.18$
\end{tabular}

*Data are mean $\left(\times 10^{-3} \mathrm{~mm}^{2} / \mathrm{s}\right) \pm$ standard deviation

$A D C=$ apparent diffusion coefficient; $F P=$ focal pancreatitis 


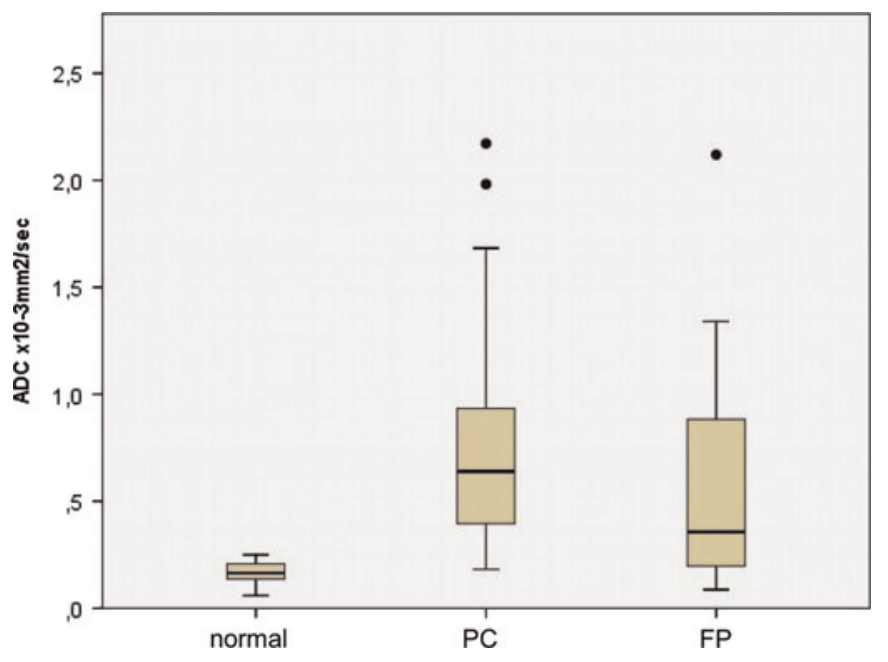

Fig. 3 Box plots of ADC values for normal pancreatic tissue, pancreatic carcinoma, and mass-forming focal pancreatitis

\section{Discussion}

Pancreatic cancer is the third most common malignancy of the gastrointestinal tract; the incidence rate is estimated at about 10 new cases per 100,000 people per year. The main task in diagnostic imaging of the pancreas is the detection and differentiation of pancreatic lesions into malignant and benign (e.g. inflammatory) entities as well as to assess resectability of pancreatic cancer. Usually morphologic changes of chronic pancreatitis result in a shrunken and atrophic pancreas, but occasionally it may present as a mass-forming FP and thus may mimic a PC (12). This may lead to a difficult preoperative differential diagnosis. Recent technical innovations have introduced diffusionweighted MR imaging with apparent diffusion coefficient (ADC) measurements. The usefulness of DWI with a singleshot spin-echo echo-planar sequence (EPI) for the evaluation of neoplastic diseases in the abdominal region has been reported by several studies $(4,8,19)$.

However, measured abdominal ADC values depend on the exact selection of $\mathrm{b}$ values, in addition ADC values also depend on the field strength of the scanner (20). There is a consistent decrease of ADC values with increased diffusion weighting as well as with increased field strength. This might partially explain the variations of ADC values

Table 2 Mann-Whitney $U$ test for differences in mean ADC values for normal pancreatic tissue, pancreatic carcinoma, and mass-forming focal pancreatitis

\begin{tabular}{|c|c|c|c|}
\hline \multirow{2}{*}{ Parameter } & \multirow[t]{2}{*}{$\begin{array}{l}\text { Sample } \\
\text { sizes }\end{array}$} & \multicolumn{2}{|c|}{$\begin{array}{c}\text { Mann-Whitney } \\
\text { U }\end{array}$} \\
\hline & & $\mathbf{U}$ & $P$ \\
\hline $\begin{array}{l}\text { Normal pancreas vs. pancreatic } \\
\text { carcinoma }\end{array}$ & $\begin{array}{l}n=17 \\
n=24\end{array}$ & 13.5 & $<0.001^{*}$ \\
\hline $\begin{array}{l}\text { Normal pancreas vs. mass-forming } \\
\text { FP }\end{array}$ & $\begin{array}{l}n=17 \\
n=20\end{array}$ & 69.5 & $0.002^{*}$ \\
\hline $\begin{array}{l}\text { Pancreatic carcinoma vs. } \\
\text { mass-forming FP }\end{array}$ & $\begin{array}{l}n=24 \\
n=20\end{array}$ & 179.0 & 0.15 \\
\hline
\end{tabular}

${ }^{*} P<0.01$

$\mathrm{FP}=$ focal pancreatitis for PC according to previous reports. Also the use of a thin slice thickness of $2.6 \mathrm{~mm}$ for the DWI imaging and the use of two $b$ values as compared to the use multiple $b$ values have influenced the ADC measurements. However, the use of a the thin slice thickness and two $b$ values did allow a rapid acquisition of images, albeit at a low signal to noise ratio (SNR) of DWI images and thus influencing the ADC values. PC has been associated with decreased ADC values compared to healthy pancreatic tissue due to the presence of fibrosis and increased cellularity which is associated with restricted water diffusion (13). Necrosis is a common feature of PC and is associated with increased ADC values due to increased motion of the water protons in the tissue (21). Inflammation within and/ or surrounding the PC is also a common feature of larger PC; this is due to the obstruction of the pancreatic duct or its side branches $(22,23)$. Inflammatory processes correlate with an increase of water diffusions, and thus have increased ADC values (14). Yoshikawa et al. found significantly increased ADC values for PC compared to ADC values for healthy pancreas parenchyma (18). Similarly, we found increased ADC values for PC and mass-forming FP compared to the pancreatic gland in the control group. This might be due to the large lesions sizes (PC and massforming FP) in our study compared to previous studies, as these larger lesions are more commonly associated with necrosis and inflammation. ADC values for the massforming FP also proved to be significantly higher compared with those of the normal pancreatic tissue. However, there was a significant overlap in ADC values for PC and massforming FP, with the consequent problem of their correct differentiation. Chronic pancreatitis can also contain areas of fibrosis and focal inflammatory reactions (12), which might explain the difficulty to differentiate these severe pancreatic lesions.

This study has some limitations, such as that the average age of the patients was significantly higher than that of our healthy controls. This might have independently influenced the measured ADC, however, the age range for the two patient groups was comparable. Second, the patient and control group were relatively small. Thus a statistically significant difference in ADC values might be established in a larger sample. An additional limitation is that we only analyzed the quantitative information of ADC maps, leaving aside the qualitative information of the obtained DWI images (24). Thus, we only evaluated the value of ADC in differentiating PC, mass-forming FP, and normal pancreas from each other. The additional information of the original images with different $b$ values has not been used and the value of DWI in the detection and differentiation has not been analyzed. Furthermore, we did not evaluate ADC values of adjacent pancreas parenchyma surrounding the lesion, which could have also yielded extra information. Finally, the ROIs to acquire ADC values covered the whole lesion including central necrosis. These larger ROIs were used, to make the method feasible in the daily clinical routine; however this might have biased the measurement of ADCs in the patient group.

In conclusion, we could not demonstrate that using ADC measurements of focal pancreatic lesions is a viable 
approach to differentiate between PC and mass-forming FP. However, a differentiation of healthy pancreatic tissue from PC and mass-forming FP was possible. Given the limited accuracy of routine MRI (T1- and T2-weighted images) for the differentiation of tumors, further studies are necessary to investigate whether DWI can improve the accuracy of MR imaging. Furthermore it remains to be seen if ADCs can be used as an imaging response criteria for therapy monitoring (5) and follow-up of palliative chemotherapy in patients with metastatic or locally advanced inoperable pancreatic cancer.

\section{Conflict of interest: None.}

\section{REFERENCES}

1 Fattahi R, Balci NC, Perman WH, et al. Pancreatic diffusion-weighted imaging (DWI): comparison between mass-forming focal pancreatitis (FP), pancreatic cancer (PC), and normal pancreas. J Magn Reson Imaging 2009;29:350-6

2 Ichikawa T, Haradome H, Hachiya J, et al. Perfusion-weighted MR imaging in the upper abdomen: preliminary clinical experience in 61 patients. Am J Roentgenol 1997;169:1061-6

3 Takahara T, Imai Y, Yamashita T, et al. Diffusion weighted whole body imaging with background body signal suppression (DWIBS): technical improvement using free breathing, STIR and high resolution 3D display. Radiat Med 2004;22:275-82

4 Taouli B, Vilgrain V, Dumont E, et al. Evaluation of liver diffusion isotropy and characterization of focal hepatic lesions with two single-shot echo-planar MR imaging sequences: prospective study in 66 patients. Radiology 2003;226:71-8

5 Chen J, Sheng J, Xing W, et al. Monitoring early response of lymph node metastases to radiotherapy in animal models: diffusion-weighted imaging vs. morphological MR imaging. Acta Radiol 2011;52:989-94

6 Boulanger Y, Amara M, Lepanto L, et al. Diffusion-weighted MR imaging of the liver of hepatitis C patients. NMR Biomed 2003;16:132-6

7 Cova M, Squillaci E, Stacul F, et al. Diffusion-weighted MRI in the evaluation of renal lesions: preliminary results. Br J Radiol 2004;77:851-7

8 Thoeny HC, Keyzer F, Oyen RH, et al. Diffusion-weighted MR imaging of kidneys in healthy volunteers and patients with parenchymal diseases: initial experience. Radiology 2005;235:911-7

9 Taouli B, Martin AJ, Qayyum A, et al. Parallel imaging and diffusion tensor imaging for diffusion-weighted MRI of the liver: preliminary experience in healthy volunteers. Am J Roentgenol 2004;183: $677-80$

10 Lemke A, Laun FB, Klauss M, et al. Differentiation of pancreas carcinoma from healthy pancreatic tissue using multiple b-values: comparison of apparent diffusion coefficient and intravoxel incoherent motion derived parameters. Invest Radiol 2009;44:769-75

11 Matsuki M, Inada Y, Nakai G, et al. Diffusion-weighed MR imaging of pancreatic carcinoma. Abdom Imaging 2007;32:481-3

12 Momtahen AJ, Balci NC, Alkaade S, et al. Focal pancreatitis mimicking pancreatic mass: magnetic resonance imaging (MRI)/magnetic resonance cholangiopancreatography (MRCP) findings including diffusion-weighted MRI. Acta Radiol 2008;49:490-7

13 Muraoka N, Uematsu H, Kimura H, et al. Apparent diffusion coefficient in pancreatic cancer: characterization and histopathological correlations. J Magn Reson Imaging 2008;27:1302-8

14 Shinya S, Sasaki T, Nakagawa Y, et al. Usefulness of diffusion-weighted imaging (DWI) for the detection of pancreatic cancer: 4 case reports. Hepatogastroenterology 2008;55:282-5

15 Takeuchi M, Matsuzaki K, Kubo H, et al. High-b-value diffusion-weighted magnetic resonance imaging of pancreatic cancer and mass-forming chronic pancreatitis: preliminary results. Acta Radiol 2008;49:383-6

16 Squillaci E, Manenti G, Cova M, et al. Correlation of diffusion-weighted MR imaging with cellularity of renal tumours. Anticancer Res 2004;24: 4175-9

17 Balci NC, Perman WH, Saglam S, et al. Diffusion-weighted magnetic resonance imaging of the pancreas. TMRI 2009;20:43-7

18 Yoshikawa T, Kawamitsu H, Mitchell DG, et al. ADC measurement of abdominal organs and lesions using parallel imaging technique. Am J Roentgenol 2006;187:1521-30

19 Chow LC, Bammer R, Moseley ME, et al. Single breath-hold diffusion-weighted imaging of the abdomen. J Magn Reson Imaging 2003;18:377-82

20 Dale BM, Braithwaite AC, Boll DT, et al. Field strength and diffusion encoding technique affect the apparent diffusion coefficient measurements in diffusion-weighted imaging of the abdomen. Invest Radiol 2010;45:104-8

21 Akisik MF, Aisen AM, Sandrasegaran K, et al. Assessment of chronic pancreatitis: utility of diffusion-weighted MR imaging with secretin enhancement. Radiology 2009;250:103-9

22 Lin A, Feller ER. Pancreatic carcinoma as a cause of unexplained pancreatitis: report of ten cases. Ann Intern Med 1990;113:166-7

23 Mujica VR, Barkin JS, Go VL. Acute pancreatitis secondary to pancreatic carcinoma. Study Group Participants. Pancreas 2000;21:329-32

24 Irie H, Kamochi N, Nojiri J, et al. High b-value diffusion-weighted MRI in differentiation between benign and malignant polypoid gallbladder lesions. Acta Radiol 2011;52:236-40 